\title{
Photodynamic Therapy with Conjugated Polymer Nanoparticles: Re- cent Advances and Therapeutic Considerations
}

\author{
Alondra M. Ortiz Ortiz ${ }^{5}$ Olivia George ${ }^{6}$, Khalaf Jasim ${ }^{1,2,7}$, Andre J. Gesquiere 1,2,3,4* \\ 'NanoScience Technology Center, University of Central Florida, Orlando, FL 32826, USA \\ ${ }^{2}$ Department of Chemistry, University of Central Florida, Orlando, FL 32816, USA \\ ${ }^{3}$ Department of Materials Science and Engineering, University of Central Florida, Orlando, FL 32816, USA \\ ${ }^{4}$ The College of Optics and Photonics (CREOL), University of Central Florida, Orlando, FL 32816, USA \\ ${ }^{5}$ Department of Mechanical Engineering, University of Puerto Rico, Mayaguez Campus, Mayaguez 00681-9000, Puerto Rico, \\ ${ }^{6}$ Department of Chemical Engineering, University of Tennessee at Chattanooga, Chattanooga, TN 37403, Tennessee \\ ${ }^{7}$ Department of Chemistry, University of Tikrit, Tikrit, Salah Al-Din PO Box 42, Iraq
}

\section{Article Info}

\section{Article Notes}

Received: April 30, 2018

Accepted: May 28, 2018

\section{*Correspondence:}

Dr. Andre J. Gesquiere

NanoScience Technology Center, University of Central

Florida, Orlando, FL 32816, USA

Email: andre@ucf.edu

(c) 2018 Gesquiere AJ. This article is distributed under the terms of the Creative Commons Attribution 4.0 International License.

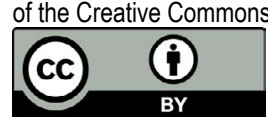

\section{ABSTRACT}

Many types of cancers require elevated levels of Reactive Oxygen Species (ROS) for uncontrolled proliferation. However, this also makes tumor cells more susceptible to ROS induced cell death by additional oxidative stress caused by external stimuli. By selectively targeting cancer cells and tumors that exhibit high levels of ROS with nanotechnology-based materials to trigger further elevation of ROS therapeutic possibilities become available. Specifically, light-activated treatment through Photodynamic Therapy (PDT) has been demonstrated as a feasible approach with Photophryn as the leading FDA approved sensitizer. However, such small molecule sensitizers do still have significant hurdles to overcome, including poor solubility, non-targeted delivery, and low absorption of light. In this mini review, recent advances in the development of Conjugated Polymer Nanoparticles (CP-NPs) solving these issues are discussed, including amplified ROS generation, tumor targeting, theranostic capabilities, and multimodal CP-NPs. We also give an outlook towards further needs in CP-NP development for clinical application.

\section{Introduction}

Reactive Oxygen Species (ROS) are essential participants for normal functioning of cells such as cell signaling and apoptosis, but have also been identified as playing a major role in promoting carcinogenesis ${ }^{1}$. The Akt pathway ensures resistance to senescence and ROS mediated apoptosis in normal cells, but in human cancers that protection is removed in order to promote uncontrolled proliferation. Thus, ROS induced apoptosis in cancer cells is facilitated compared to normal cells ${ }^{2,3}$. This double-edged sword for human cancer growth and proliferation opens avenues to selectively target and eliminate cancer cells and tumors that exhibit high levels of ROS through Photodynamic Therapy (PDT).

Attempts to utilize this mechanism in therapeutic approaches have been reported based on inorganic (quantum dots, metal oxide nanoparticles) and metal nanoparticles (gold, silver, and iron) ${ }^{4,5}$. In these instances intracellular damage and cell death typically occurred due to lipid peroxidation of mitochondria. However, the lack of biocompatibility of these nanomaterials is of concern, and in some cases such as quantum dots there is known cytotoxicity. It is thus unlikely that these approaches will lead to commercial products and therapies. 
Distinct advantages of CP-NPs include tunable surface functionalization, minimal need for purification, biocompatibility and buffer dispersibility. There are some disadvantages, however, such as that the particles could disintegrate before reaching the target since they are not cross-linked, and that the PDT photochemistry bleaches the particles i.e. no longer trackable by fluorescence imaging.

Biophotonics based on novel nanoparticle designs has brought significant advances to sensing, imaging, and therapeutic modalities for biological applications. Recently, there has been a surge in the development of organic polymer-based nanoparticles as PDT sensitizers. McNeil's group was at the forefront in reporting conjugated polymer nanoparticles (CP-NPs) for bioimaging and PDT applications, ${ }^{6}$ which was extended by our and other groups into CP-NPs with multimodal capabilities. These advances will be discussed in this mini review.

\section{CP-NPs Preparation Requires Minimal Chemistry}

The reprecipitation method has recently become the most commonly used method for CP-NPs fabrication, ${ }^{7,8}$ although laser ablation of microcrystals has also made a significant impact on the field ${ }^{9}$. Both methods are illustrated in Figure 1. The straightforward fabrication of CP-NPs by the reprecipitation method is attractive since it is a simple solution-processing technique in which a solution of organic material in good solvent is injected into a very poor solvent for the organic material. By managing solvent types and starting concentration of the conjugated polymer stock solutions it is also possible to gain control over particle size (Figure 1). The reprecipitation method finds its origin in the preparation of dye molecule nanoparticles ${ }^{10}$. Upon injection, a stable suspension of nanoparticles is formed due to hydrophobic aggregation of the organic compounds when entering the water phase. Zeta potential measurements for CP-NPs typically reveal a slightly negative charge that stabilizes the hydrophobic CPNPs in water and buffer suspension when the CP-NPs have no additional surface coating ${ }^{11}$. Surface modification of the CP-NPs provides further control on their surface charge ${ }^{12}$. This can be accomplished through careful design of the polymers ${ }^{12-15}$ or by application of mild chemistry to surface functional groups, which is further discussed below (vide infra). Particle size, hydrophobicity, and surface charge directly affect quantity and internalization mechanism of CP-NPs by cancer cells ${ }^{12,16-18}$.

\section{Mechanism of ROS formation and PDT effects}

ROS are formed during the native function of cells. During the oxidative metabolism in mitochondria, single electron reduction of molecular oxygen to superoxide anion occurs in the cell body ${ }^{19}$. Other cell organelles such as the endoplasmic reticulum and the nuclear membrane also have electron transport systems that lead to formation of $\operatorname{ROS}^{20}$.

Similar mechanisms for ROS formation are at play when exogenous ROS sensitizers are introduced for PDT. By providing a light source to the PDT sensitizer that matches its absorption spectrum the photosensitizer will enter the singlet exited state ${ }^{14,21}$. From there either relaxation to the ground state or intersystem crossing to the triplet state occurs. The latter is desired for PDT, especially for conjugated polymer sensitizers, because of the similarity of their triplet energy with the excited state of oxygen (singlet oxygen). This feature enables both efficient energy and charge transfer processes towards formation of ROS

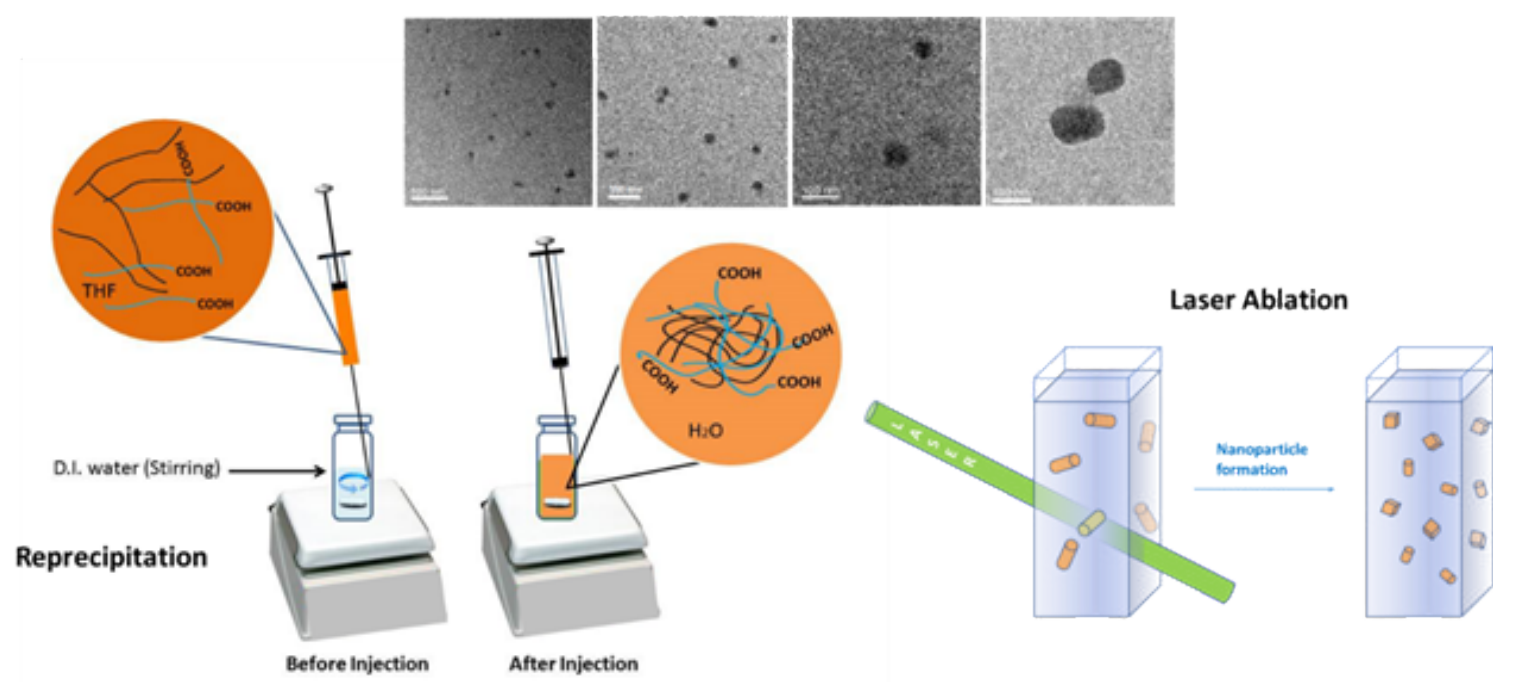

Figure 1: Schematic representation of the reprecipitation and laser ablation methods for CP-NPs preparation. In the reprecipitation method, surface modalities can be introduced by co-precipitation. The TEM images in the upper section show the possibility for size control (unpublished results). Scale bar is $100 \mathrm{~nm}$. 


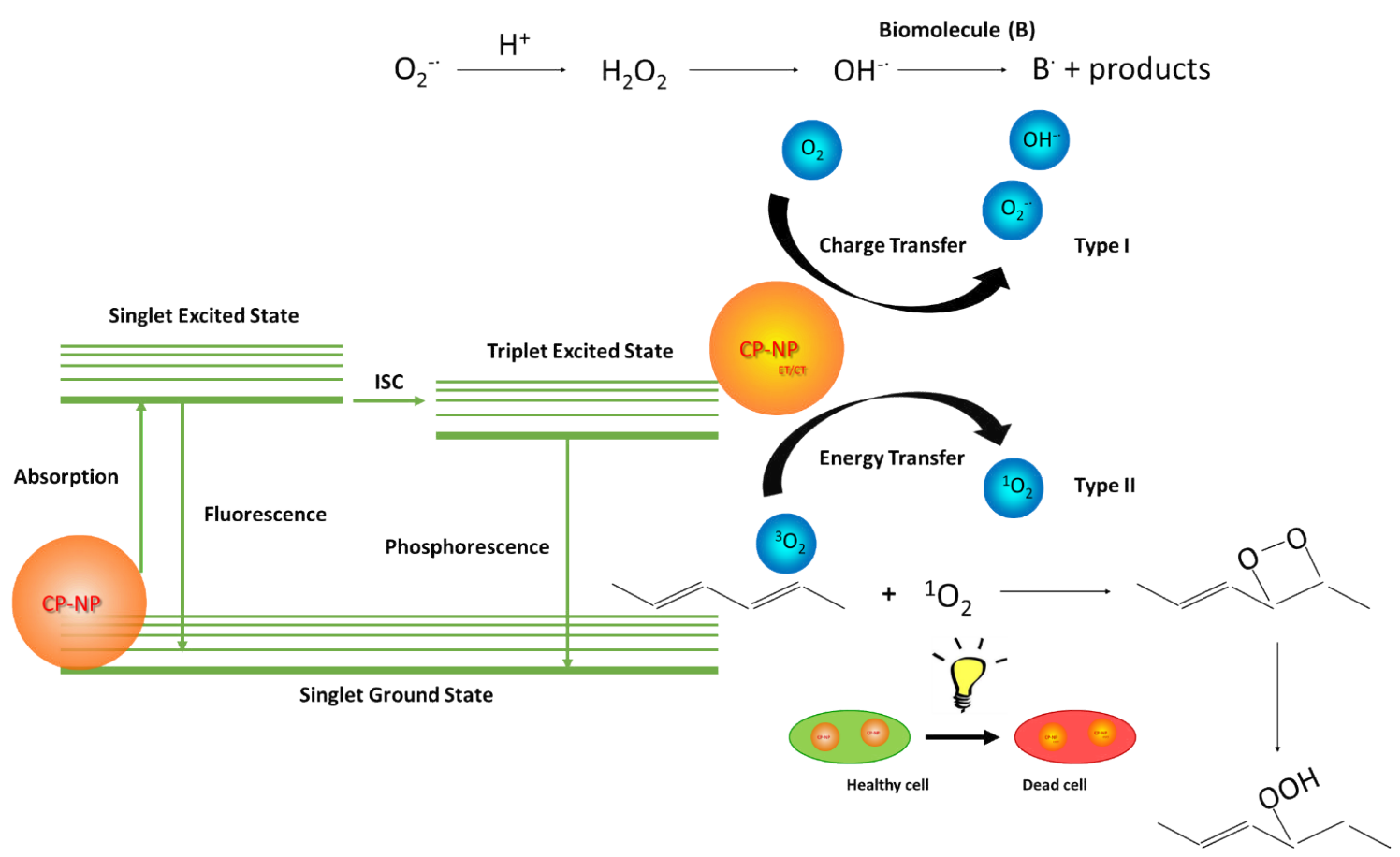

Figure 2: Diagram of Photodynamic Therapy with CP-NPs as photosensitizer. Once the CP-NPs reach the triplet excited state charge transfer to oxygen or biomolecules occurs (Type I mechanism) or energy transfer takes place to form singlet oxygen (Type II mechanism). Either mechanism leads to elevated ROS under illumination and potential for therapeutics.

that can be used therapeutically in cancer treatment ${ }^{22-25}$. There are two well-known pathways for ROS formation ${ }^{21,26}$. In the type I mechanism, charge transfer occurs from the triplet state of the photosensitizer to oxygen to produce a superoxide radical anion. This mechanism also works with biomolecules and solvent molecules such as those found in cells. In the type II mechanism, energy transfer occurs from the triplet state of the photosensitizer to oxygen to yield singlet oxygen ${ }^{14,25,27,28}$. The resulting elevated levels of ROS induce oxidative stress that lead to cell damage and cell death (Figure 2) ) $^{8,14,23,25-27}$.

\section{CP-NPs Photophysics Enables Enhanced PDT Properties}

Conjugated polymers are multichromophoric systems that typically contain a few tens to over a hundred chromophores depending on molecular weight and have well-known singlet and triplet properties. Their corresponding nanoparticles contain from a few to several tens of polymer chains. Considering these properties, it becomes clear why CP-NPs are significant as a next generation of PDT sensitizers. CP-NPs have major advantages over current small molecule sensitizers (including FDA approved ones) given the CP-NPs' (i) large extinction coefficients $\left(\varepsilon>10^{\wedge} 7 \mathrm{~L} \mathrm{~mol}^{-1} \mathrm{~cm}^{-1}\right)$, (ii) efficient triplet formation as necessary for PDT, with triplet energies that are close to that of oxygen, ${ }^{29}$ (iii) intrinsic ROS formation (no need for sensitizing dopants or encapsulation of conventional sensitizers), (iv) low to absent cytotoxicity found in vitro (in dark), (v) buffer dispersibility and stability, (vi) intrinsic fluorescence for tracking of delivery (no need for dye dopant or attachment), and (vii) adaptable design for targeted delivery. The large extinction coefficient $\varepsilon$ indicates that the photon absorption rate is 2 to 3 orders of magnitude higher than current small molecule sensitizers such as Phthalocyanine, Porphyrine, and Photophryn (where the best sensitizers have an extinction coefficient of several $10^{\wedge} 5$ at best). These properties result in triplet generation rates (through intersystem crossing, ISC) that are 2 to 3 orders of magnitude greater than those reported for current small molecule sensitizers. The intrinsic fluorescence has been an additional attractive feature since it allows for tracking of CP-NPs in-vitro and in-vivo by optical imaging ${ }^{22,24}$. Reports have also started appearing that investigate the tunability of CP-NPs' optical properties $^{27}$.

The development of blended CP-NPs has in some cases lead to additional improvement in singlet oxygen or radical anion formation. In such cases, the CP-NPs contain additional small molecule photosensitizers. Amplification of singlet oxygen generation from CP-NPs has recently been reported by the Palacios group ${ }^{25,28}$. The authors reported that poly(9,9-dioctylfluorene-alt-benzothiadiazole) (F8BT) doped with platinum octaethylporphyrin (PtOEP) produces singlet oxygen from F8BT rather than from 
PtOEP. The dopant serves to increase the quantum yield of singlet oxygen formation through the intricacies of conjugated polymer photophysics. Downconversion has also been employed by several groups to enhance singlet oxygen generation. In this CP-NP design, photosensitizer dopants are loaded inside the CP-NPs. The CP-NPs then act as antenna systems for the PDT sensitizer. After light absorption by the CP-NPs, energy is transferred by FRET to the triplet sensitizer, either in a single step or in a multistep energy transfer cascade. This approach has been completed for different conjugated polymer-sensitizer combination, including for Rose Bengal molecules attached on the surface of coumarin 153-dye-doped poly[N-vinyl carbazole], Rose Bengal and Rose Bengal Methyl Ester blended with poly[(9,9-dioctylfluorenyl-2,7diyl)-alt-co-(1,4-benzo- $\{2,10,3\}$-thiadiazole $)]$, poly[(9,9dioctylfluorenyl-2,7-diyl)-alt-co-(1,4-benzo- $\left\{2,1^{\prime}, 3\right\}$ thiadiazole)] blended with porphyrin, and for the conjugated polymers conjugated polymers poly(9,9-dihexylfluorene), poly(9,9-dioctylfluorenyl-2,7-diyl) and poly[\{9,9-dioctyl2,7-divinylene-fluoreneylene\}-alt-co-\{2-methoxy-5-(2ethylhexyloxy)-1,4-phenylene\}] (PFBT) blended with Rose Bengal or porphyrin for instance ${ }^{6,30-34}$. With this approach, sensitizers that are not buffer compatible can be solubilized in aqueous media for increased bioavailability while enhancing their performance. A charge transfer cascade was reported by our group through the use of fullerene dopants loaded into (poly[2-methoxy-5-(2-ethylhexyloxy)-p-phenylenevinylene] (MEH-PPV) CP-NPs ${ }^{35}$. Besides enhanced PDT effect, an interesting observation was the absence of fullerene-induced cytotoxicity, suggesting that encapsulation in CP-NPs can increase biocompatibility of PDT sensitizers ${ }^{36}$.

\section{Multimodal CP-NPs: Tumor Targeting and Multifunctional Designs}

Targeted delivery is an important consideration for therapeutic impact and clinical relevance. For CP-NPs the constituent polymer usually does not have functional groups that allow for easy ligand binding chemistry. A copolymer with for example carboxyl or amine moieties is often used to allow for targeting ligand attachment. Once the CP-NPs reach the target site and accumulate, typically by EPR effect, the CP-NPs undergo receptor-mediated endocytosis. This approach has been demonstrated for small molecule ligands and peptides ${ }^{23,37}$, which promises advanced therapeutic applications of targeted CP-NPs. The $\mathrm{Xu}$ group recently reported significant advances using peptide functionalized MEH-PPV CP-NPs for in-vivo theranostics of triple-negative breast cancer $^{38}$.

Multimodality has also received a lot of attention in the last few years. There has been a major increase in work reported on novel nanoparticles that have multiple imaging, sensing or therapeutic modalities, including combinations thereof. This also holds true for CP-NPs. Multifunctional CP-NPs that are tumor targeted, fluorescent, and are able to deliver combined PDT and photothermal therapy (PTT) were reported recently, developed from the conjugated polymers poly[9,9-bis(2-(2-(2-methoxyethoxy) ethoxy)-ethyl)fluorenyldivinylen e]-alt-4,7-(2,1,3benzothiadiazole) (PFVBT) and poly[(4,4,9,9-tetrakis(4(octyloxy)phenyl)-4,9-dihydro-s-indacenol-dithiophene2,7-diyl)-alt-co-4,9-bis(thiophen-2-yl)-6,7-bis (4(hexyloxy)phenyl)-thiadiazolo-quinoxaline] (PIDTTTQ), and from D-A-type alkyl-chain-grafted bithiophene(3E,7E)-3,7-bis(2-oxoindolin-3-ylidene)benzo-[1,2-b:4,5$\left.\mathrm{b}^{\prime}\right]$-difuran-2,6(3H,7H)-dione $\mathrm{e}^{39,40}$. At the basis of the design are CP-NPs with strong near-infrared absorption, which will be discussed further below (vide infra). Multimodality through combined imaging and biosensing, including $\mathrm{pH}$, gas, ion, and temperature sensing, has also been investigated extensively for CP-NPs ${ }^{41,42}$. Novel therapeutics have been incorporated into CP-NPs by the Moon group specially for gene delivery ${ }^{43,44}$. The investigators applied their multimodal CP-NPs as imaging and siRNA delivery vehicles.

\section{In-vivo application of CP-NPs: Looking Ahead}

Substantial progress has been made to bring CPNPs towards clinical relevance, but further advances are required. These include the development of near-infrared (NIR) absorbing/emitting nanoparticles and in vivo studies on CP-NPs. So far, donor-acceptor conjugated polymers were included in CP-NPs for synergistic PDT and PTT under NIR excitation and detection ${ }^{40}$. An NIR theranostic approach was also reported in which NIR tracking of poly(1,2-bis(4((6-bromohexyl)oxy)phenyl)-1,2-diphenylethene-coalt-9,10-anthraquinone) CP-NPs was followed by PDT ${ }^{45}$. In-vivo targeted delivery, tracking and therapeutics have been shown as well. Brain tumor targeted PFBT CP-NPs exhibited accumulation at the tumor site in genetically engineered mouse models ${ }^{46}$. Similar observations were made for subcutaneous RCC (human renal cell carcinoma) xenografts ${ }^{24}$. PFBT CP-NPs were applied in vivo with human gastric adenocarcinoma xenografts, and a comparison between intravenous and intratumoral injection was made. Significant tumor inhibition and eradication were observed, respectively ${ }^{34}$. SKBR-3 breast cancer tumorbearing mice were treated with anti-HER2 functionalized NIR absorbing PFVBT/PIDTTTQ CP-NPs.[39] Excellent tumor targeting and accumulation of the anti-HER2 CPNPs at the tumor site were observed. Synergistic PDT/PTT showed near complete inhibition of tumor growth.

Current advances in multiphoton excitation and bioluminescence energy transfer (BRET) capabilities for CP-NPs can lead to even further progress. Multiphoton excitation allows the use of NIR light sources to activate visible absorbing PDT sensitizers. This approach has 
been successful for a variety of CP-NPs designs, including tumor targeted and activatable CP-NPs with high twophoton cross sections such as from poly\{9,9-bis[6"(bromohexyl)-fluorene-2,7-ylenevinylene]-co-alt-1,4(2,5-dicyanophenylene)\} as conjugated polymers ${ }^{6,47-49}$. Antitumor activity of BRET-based CP-NPs where luminol was incorporated as a bioluminescence precursor was studied. An over 50\% tumor inhibition ratio was found for HeLa (cervical cancer) tumor bearing nude mice ${ }^{50}$. Similarly, strong tumor growth inhibition was found for luciferase modified BRET CP-NPs applied in vivo to breast cancer (MCF-7) and cervical cancer (HeLa) tumor model ${ }^{551}$. These BRET systems have demonstrated clear promise in deep tissue imaging and light activated tumor treatment. Near-infrared optical properties, multiphoton excitation and BRET will be important factors in the success of CPNPs as high impact anticancer theranostics.

\section{Conclusion}

In recent years, CP-NPs have developed from a novelty in bioimaging to a full-fledged multimodal platform for nanomedicine. At present, CP-NPs are tumor targeted, brightly fluorescent, and capable of intracellular sensing and cargo delivery. The future looks even brighter with the advent of near-infrared abilities for both imaging and therapeutics, including through multiphoton excitation, and the use of bioluminescent materials in CP-NPs as builtin light sources for BRET schemes. These developments are bringing CP-NPs into the NIR I biological window, which opens up avenues for deeper tissue in vivo theranostics and a positive outlook for clinical viability.

\section{Acknowledgments}

The authors would like to thank the National Science Foundation for support of this work through REU site EEC 1560007.

\section{Conflict of Interest Statement}

The authors have no conflict of interest to disclose.

\section{References}

1. Liou GY, Storz P. Reactive oxygen species in cancer. Free Radic Res. 44: 479-496.

2. Nogueira V, Park Y, Chen CC, et al. Akt Determines Replicative Senescence and Oxidative or Oncogenic Premature Senescence and Sensitizes Cells to Oxidative Apoptosis. Cancer Cell. 2008; 14: 458-470.

3. Huang P, Trachootham D, Alexandre J. Targeting cancer cells by ROSmediated mechanisms: a radical therapeutic approach. Nat Rev Drug Discov. 2009; 8: 579-591.

4. Managa M, Britton J, Prinsloo E, et al. Effects of pluronic silica nanoparticles on the photophysical and photodynamic therapy behavior of triphenyl-p-phenoxy benzoic acid metalloporphyrins. Journal of Coordination Chemistry. 2016; 69: 3491-3506.

5. Lee M, Lee H, Rao NV, et al. Gold-stabilized carboxymethyl dextran nanoparticles for image-guided photodynamic therapy of cancer. Journal of Materials Chemistry B. 2017; 5: 7319-7327.
6. Grimland JL Wu C, Ramoutar RR, et al. Photosensitizer-doped conjugated polymer nanoparticles with high cross-sections for oneand two-photon excitation. Nanoscale. 2011; 3: 1451-1455.

7. Wu C, Bull B, Szymanski C, et al. Multicolor conjugated polymer dots for biological fluorescence imaging. ACS nano. 2008; 2: 2415-2423.

8. Doshi M, Gesquiere AJ. Photodynamic Therapy with Blended Conducting Polymer/Fullerene Nanoparticle Photosensitizers. Journal of visualized experiments : JoVE. 2015; 104: e53038.

9. Asahi T, Sugiyama T, Masuhara H. Laser fabrication and spectroscopy of organic nanoparticles. Accounts of chemical research. 2008; 41: 1790-1798.

10. Kasai H, Nalwa HS, Oikawa H, et al. A novel preparation method of organic microcrystals. Japanese Journal of Applied Physics. 1992; 31: L1132.

11. Doshi M, Copik A, Gesquiere AJ. Development and characterization of conducting polymer nanoparticles for photodynamic therapy in vitro. Photodiagnosis and photodynamic therapy. 2015; 12: 476-489.

12. He C Hu Y, Yin L, et al. Effects of particle size and surface charge on cellular uptake and biodistribution of polymeric nanoparticles. Biomaterials. 2010; 31: 3657-3666.

13. Yu CY, Godana AS. Conjugated polymer nanoparticles based on fluorenes, PEGylated carbazoles and diphenylamines. European Polymer Journal. 2018; 99: 165-171.

14. Zaquen N, Lutsen L, Vanderzande D, et al. Controlled/living polymerization towards functional poly ( $\mathrm{p}$-phenylene vinylene) materials. Polymer Chemistry. 2016; 7:1355-1367.

15. Zaquen N, Verstraete K, Lutsen L, et al. Modifiable poly (p-phenylene vinylene) copolymers towards functional conjugated materials. Polymer Chemistry. 2016; 7: 4771-4781.

16. Abou Matar T, Karam P. The Role of Hydrophobicity in the Cellular Uptake of Negatively Charged Macromolecules. Macromolecular bioscience. 2018; 18: 1700309.

17. Lee J, Twomey M, Machado C, et al. Caveolae-Mediated Endocytosis of Conjugated Polymer Nanoparticles. Macromolecular bioscience. 2013; 13: 913-920.

18. Peters M, Zaquen N, D'Olieslaeger L, et al. PPV-based conjugated polymer nanoparticles as a versatile bioimaging probe: A closer look at the inherent optical properties and nanoparticle-cell interactions. Biomacromolecules. 2016; 17: 2562-2571.

19. Fariss MW, Chan CB, Patel M, et al. Defining mitochondrial targets to combat the pleiotropic effects of toxic oxidative stress. Mol Interv. 2005; 5: 94-111.

20. Hancock JT, Desikan R, Neill SJ. Role of reactive oxygen species in cell signalling pathways. Biochem Soc Trans. 2001; 29: 345-350.

21. Mehraban N, Freeman HS. Developments in PDT sensitizers for increased selectivity and singlet oxygen production. Materials. 2015; 8: 4421-4456.

22. Braeken Y, Cheruku S, Ethirajan A, et al. Conjugated Polymer Nanoparticles for Bioimaging. Materials. 2017; 10: 1420.

23. Doshi M, Krienke M, Khederzadeh S, et al. Conducting polymer nanoparticles for targeted cancer therapy. RSC Advances. 2015; 5: 37943-37956.

24. Pu K, Shuhendler AJ, Valta MP, et al. Phosphorylcholine-Coated Semiconducting Polymer Nanoparticles as Rapid and Efficient Labeling Agents for In Vivo Cell Tracking. Advanced healthcare materials. 2014; 3: 1292-1298.

25. Spada RM, Macor LP, Hernández LI, et al. Amplified singlet oxygen generation in metallated-porphyrin doped conjugated polymer nanoparticles. Dyes and Pigments. 2018; 149: 212-223. 
26. Meng Z, Hou W, Zhou H, et al. Therapeutic Considerations and Conjugated Polymer-Based Photosensitizers for Photodynamic Therapy. Macromolecular rapid communications. 2018; 39: 1700614

27. D’Olieslaeger L Braeken Y, Cheruku S, et al. Tuning the optical properties of poly ( $p$-phenylene ethynylene) nanoparticles as bioimaging probes by side chain functionalization. Journal of colloid and interface science. 2017; 504: 527-537.

28. Ibarra LE Porcal GV, Macor LP, et al. Metallated porphyrin-doped conjugated polymer nanoparticles for efficient photodynamic therapy of brain and colorectal tumor cells. Nanomedicine. 2018; 13: 605624

29. Yu J, Lammi R, Gesquiere AJ, et al. Singlet- triplet and triplet- triplet interactions in conjugated polymer single molecules. The Journal of Physical Chemistry B. 2005; 109: 10025-10034.

30. Bhattacharyya S, Barman MK, Baidya A, et al. Singlet Oxygen Generation from Polymer Nanoparticles-Photosensitizer Conjugates Using FRET Cascade. The Journal of Physical Chemistry C. 2014; 118: 9733-9740.

31. Chang K, Tang Y, Fang X, et al. Incorporation of porphyrin to $\pi$-conjugated backbone for polymer-dot-sensitized photodynamic therapy. Biomacromolecules. 2016; 17: 2128-2136.

32. Haimov E, Weitman H, Ickowicz D, et al. Pdots nanoparticles load photosensitizers and enhance efficiently their photodynamic effect by FRET. RSC Advances. 2015; 5: 18482-18491.

33. He $\mathrm{R}, \mathrm{Hu} \mathrm{M}, \mathrm{Xu} \mathrm{T}$, et al. Conjugated copolymer-photosensitizer molecular hybrids with broadband visible light absorption for efficient light-harvesting and enhanced singlet oxygen generation. Journal of Materials Chemistry C. 2015; 3: 973-976.

34. Li S, Chang K, Sun K, et al. Amplified singlet oxygen generation in semiconductor polymer dots for photodynamic cancer therapy. ACS applied materials \& interfaces. 2015; 8: 3624-3634.

35. Doshi M, Treglown K, Copik A, et al. Composite conjugated polymer/ fullerene nanoparticles as sensitizers in photodynamic therapy for cancer. BioNanoScience. 2014; 4: 15-26.

36. Goodarzi S, Da Ros T, Conde J, et al. Fullerene: Biomedical engineers get to revisit an old friend. Materials Today. 2017; 20 : 460-480.

37. Feng L, Zhu J, Wang Z. Biological functionalization of conjugated polymer nanoparticles for targeted imaging and photodynamic killing of tumor cells. ACS applied materials \& interfaces. 2016; 8: 1936419370.

38. Jin G, He R, Liu Q, et al. Theranostics of Triple-Negative Breast Cancer Based on Conjugated Polymer Nanoparticles. ACS applied materials \& interfaces. 2018; 10: 10634-10646.
39. Feng G, Fang Y, Liu J, et al. Multifunctional Conjugated Polymer Nanoparticles for Image-Guided Photodynamic and Photothermal Therapy. Small. 2017; 13: 1602807.

40. Yang T, Liu L, Deng Y, et al. Ultrastable Near-Infrared ConjugatedPolymer Nanoparticles for Dually Photoactive Tumor Inhibition. Advanced Materials. 2017; 29: 1700487.

41. Lyu Y, Pu K. Recent advances of activatable molecular probes based on semiconducting polymer nanoparticles in sensing and imaging. Advanced Science. 2017; 4: 1600481.

42. Sun J, Ling P, Gao F. A Mitochondria-Targeted Ratiometric Biosensor for $\mathrm{pH}$ Monitoring and Imaging in Living Cells with Congo-RedFunctionalized Dual-Emission Semiconducting Polymer Dots. Analytical chemistry. 2017; 89: 11703-11710.

43. Moon JH. Conjugated Polymers for Gene Delivery. Conjugated Polymers for Biological and Biomedical Applications. 2018; 215-241.

44. Moon JH, Mendez E, Kim Y, et al. Conjugated polymer nanoparticles for small interfering RNA delivery. Chemical Communications. 2011; 47: 8370-8372.

45. Wu W, Feng G, Xu S, et al. A photostable far-red/near-infrared conjugated polymer photosensitizer with aggregation-induced emission for image-guided cancer cell ablation. Macromolecules. 2016; 49: 5017-5025.

46. Wu C, Hansen SJ, Hou Q et al. Design of highly emissive polymer dot bioconjugates for in vivo tumor targeting. Angewandte Chemie International Edition. 2011; 50: 3430-3434.

47. Cepraga C, Marotte S, Ben Daoud E, et al. Two-Photon PhotosensitizerPolymer Conjugates for Combined Cancer Cell Death Induction and Two-Photon Fluorescence Imaging: Structure/Photodynamic Therapy Efficiency Relationship. Biomacromolecules. 2017; 18: 4022-4033.

48. Gangopadhyay M, Mukhopadhyay SK, Gayathri S, et al. Fluorenemorpholine-based organic nanoparticles: lysosome-targeted $\mathrm{pH}$ triggered two-photon photodynamic therapy with fluorescence switch on-off. Journal of Materials Chemistry B. 2016; 4: 1862-1868.

49. Shen X, Li S, Li L, et al. Highly Efficient, Conjugated-Polymer-Based Nano-Photosensitizers for Selectively Targeted Two-Photon Photodynamic Therapy and Imaging of Cancer Cells. Chemistry-A European Journal. 2015; 21: 2214-2221.

50. Yuan H, Wang B, Lv F, et al. Conjugated-Polymer-Based EnergyTransfer Systems for Antimicrobial and Anticancer Applications. Advanced Materials. 2014; 26: 6978-6982.

51. Yang Y, Hou W, Liu S, et al. Biodegradable Polymer Nanoparticles for Photodynamic Therapy by Bioluminescence Resonance Energy Transfer. Biomacromolecules. 2017; 19. 201-208. 